\title{
Long-Time Behaviours of a Stochastic Predator-Prey System with Holling-Type II Functional Response and Regime Switching
}

\author{
Weili Kong (iD) and Yuanfu Shao $\mathbb{1 D}^{2}$ \\ ${ }^{1}$ College of Teacher Education, Qujing Normal University, Qujing, Yunnan 655011, China \\ ${ }^{2}$ College of Science, Guilin University of Technology, Guilin, Guangxi 541004, China \\ Correspondence should be addressed to Yuanfu Shao; shaoyf0829@163.com
}

Received 6 December 2020; Revised 22 January 2021; Accepted 2 February 2021; Published 15 February 2021

Academic Editor: Stanislaw Migorski

Copyright (c) 2021 Weili Kong and Yuanfu Shao. This is an open access article distributed under the Creative Commons Attribution License, which permits unrestricted use, distribution, and reproduction in any medium, provided the original work is properly cited.

Considering the impacts of white noise, Holling-type II functional response, and regime switching, we formulate a stochastic predator-prey model in this paper. By constructing some suitable functionals, we establish the sufficient criteria of the stationary distribution and stochastic permanence. By numerical simulations, we illustrate the results and analyze the influence of regime switching on the dynamics.

\section{Introduction}

Functional responses are very important in the predatorprey system, which is the amount of prey catch per predator per unit of time and has significant effect on the dynamical properties. Usually there are two kinds of functional response: prey dependent (such as Holling II and Holling IV, see [1-3]) and predator dependent (such as Hassell-Varley, Beddington-DeAngelis, and Crowley-Martin, see [4, 5]). Recently, a number of researchers have devoted their efforts to the predator-prey system with functional response and obtained some nice results [1-7].

For the ecological system, the growth rate of population is inevitably affected by environmental white noise, which almost exists everywhere in real world [8-10]. May reveals that due to stochastic fluctuations in environmental conditions, all the natural parameters exhibit a certain amount of random perturbations, and hence, random disturbance is introduced in many mathematical models to reveal the effect of white noise [10-15]. Besides the white noise, the growth of species also suffers from fluctuating environments such as hurricanes and earthquakes, which is described by colorful noise in mathematical modelling [16-18]. The colorful noise may take several values and switch among different regimes of environments. The switching is memoryless, and the waiting time for the next switching follows an exponential distribution. That is, in mathematical sense, it is a Markovian process. Actually, when the environments fluctuate frequently, colorful noise may bring great influence to population dynamics and even change the permanence and extinction of species, so the impacts of colorful noise on population dynamics have attracted many researchers, see, e.g., [19-22].

Motivated by above discussion, in this article, we formulate a stochastic model with Holling-type II functional response and colorful noise. By stochastic analysis, we aim to study the stability in distribution and stochastic permanence of the system.

The rest of this paper is structured as follows. Section 2 begins with our model and some notations. Section 3 is devoted to the stability in distribution of the above system. Section 4 focuses on the stochastic permanence. Some examples are given to illustrate our main results in Section 5. Finally, a brief conclusion and discussion are given to end the paper in Section 6.

\section{The Model and Notations}

Hsu and Huang [6] proposed the following predator-prey model with Holling-type II functional response: 


$$
\left\{\begin{array}{l}
\mathrm{d} x(t)=x(t)\left(r_{1}-b_{1} x(t)-\frac{c_{1} y(t)}{1+x(t)}\right) \mathrm{d} t+\sigma_{1} x(t) \mathrm{d} B_{1}(t) \\
\mathrm{d} y(t)=y(t)\left(-r_{2}-b_{2} y(t)+\frac{c_{2} x(t)}{1+x(t)}\right) \mathrm{d} t+\sigma_{2} y(t) \mathrm{d} B_{2}(t)
\end{array}\right.
$$

where $r_{1}>0$ and $-r_{2}<0$ represent the birth rate of prey and death rate of predator, respectively; $b_{1}$ and $b_{2}$ are intraspecific competition rate between species; $c_{1}>0$ is the capture rate, and $c_{2}>0$ is the conversion rate of food; $\sigma_{i}^{2}(i=$ $1,2)$ denotes the density of white noise; $(y(t) / 1+x(t))$ is the Holling-type II functional response. $B_{1}(t)$ and $B_{2}(t)$ are independent standard Brownian motions defined on the probability space $\left(\Omega, \mathscr{F},\left\{\mathscr{F}_{t}\right\}_{t \geq 0}, P\right)$ with a filtration $\left\{\mathscr{F}_{t}\right\}_{t \geq 0}$ satisfying the usual conditions (i.e., it is right continuous and $\mathscr{F}_{0}$ contains all $p$-null set). In view of the impact of regime switching (colorful noise) analyzed before, system (1) turns to the following:

$$
\left\{\begin{array}{l}
\mathrm{d} x(t)=x(t)\left(r_{1}(\alpha(t))-b_{1}(\alpha(t)) x(t)-\frac{c_{1}(\alpha(t)) y(t)}{1+x(t)}\right) \mathrm{d} t+\sigma_{1}(\alpha(t)) x(t) \mathrm{d} B_{1}(t), \\
\mathrm{d} y(t)=y(t)\left(-r_{2}(\alpha(t))-b_{2}(\alpha(t)) y(t)+\frac{c_{2}(\alpha(t)) x(t)}{1+x(t)}\right) \mathrm{d} t+\sigma_{2}(\alpha(t)) y(t) \mathrm{d} B_{2}(t) .
\end{array}\right.
$$

The regime switching $\alpha(t)$ is a Markovian chain in a finite state space $\mathbb{S}=\{1,2, \ldots, N\}$. The generator of $\alpha(t)$ is defined as $\chi=\left(\chi_{i j}\right)_{N \times N}$ with

$$
P\left\{\alpha(t+\epsilon=j \mid \alpha(t)=i\}= \begin{cases}\chi_{i j} \epsilon+o(\epsilon), & i \neq j, \\ 1+\chi_{i i} \epsilon+o(\epsilon), & i=j,\end{cases}\right.
$$

where $\epsilon>0, \chi_{i j}$ is the transition rate from the $i$ th stage to the $j$ th stage and $\chi_{i j} \geq 0$ if $i \neq j$ while $\chi_{i i}=-\sum_{i \neq j} \chi_{i j}$. It is often assumed that every sample of $\alpha(t)$ is a right continuous step function and irreducible with a finite simple jumps in any finite subinterval of $R_{+}=[0, \infty)$. It obeys a unique stationary distribution $\pi=\left(\pi_{1}, \pi_{2}, \ldots, \pi_{N}\right)$ satisfying $\pi \chi=0$ and $\sum_{k=1}^{N} \pi_{k}=1, \pi_{k}>0, \forall k \in \mathbb{S}$. The detailed switching mechanism of the hybrid system is referred to [19, 23].

Let $u(t)=\ln x(t), v(t)=\ln y(t)$, then system (2) is equivalent to the following model:

$$
\left\{\begin{array}{l}
\mathrm{d} u(t)=\left(r_{1}(\alpha(t))-\frac{\sigma_{1}^{2}(\alpha(t))}{2}-b_{1}(\alpha(t)) e^{u(t)}-\frac{c_{1}(\alpha(t)) e^{v(t)}}{1+e^{u(t)}}\right) \mathrm{d} t+\sigma_{1}(\alpha(t)) \mathrm{d} B_{1}(t), \\
\mathrm{d} v(t)=\left(-r_{2}(\alpha(t))-\frac{\sigma_{2}^{2}(\alpha(t))}{2}-b_{2}(\alpha(t)) e^{v(t)}-\frac{c_{2}(\alpha(t)) e^{u(t)}}{1+e^{u(t)}}\right) \mathrm{d} t+\sigma_{2}(\alpha(t)) \mathrm{d} B_{2}(t) .
\end{array}\right.
$$

For the later discuss, we introduce some notations about the Itô's integral for stochastic differential equations with Markovian switching $[19,22]$. Let

$$
\mathrm{d} x(t)=f(x(t), t, \alpha(t)) \mathrm{d} t+g(x(t), t, \alpha(t)) \mathrm{d} B(t),
$$

where $f: R^{2} \times R_{+} \times \mathbb{S} \longrightarrow R^{2}, g: R^{2} \times R_{+} \times \mathbb{S} \longrightarrow R^{2 \times 2}$ are measurable functions. Let $V \in C^{2,1}\left(R^{2} \times R_{+} \times \mathbb{S}, R^{2}\right)$. Define the operator $L V$ as follows:

$$
\begin{aligned}
& L V(x, t, k)=V_{t}(x, t, k)+V_{x}(x, t, k) f(x, t, k) \\
& +\frac{1}{2} \operatorname{trace}\left[g^{T}(x, t, k) V_{x x}(x, t, k) g(x, t, k)\right]+\sum_{j=1}^{N} \chi_{k j} V(x, t, j),
\end{aligned}
$$

where $\quad V_{t}(x, t, k)=(\partial V(x, t, k) / \partial t), \quad V_{x}(x, t, k)=((\partial V$ $\left.\left.(x, t, k) / \partial x_{1}\right),\left(\partial V(x, t, k) / \partial x_{2}\right)\right), \quad$ and $\quad V_{x x}(x, t, k)=$ $\left(\partial^{2} V(x, t, k) / \partial x_{i} \partial x_{j}\right)_{2 \times 2}, i, j=1,2$. 
The generalized Itô's formula is defined as

$\mathrm{d} V(x, t, k)=L V(x, t, k) \mathrm{d} t+V_{x}(x, t, k) g(x, t, k) \mathrm{d} B(t)$.

Lemma 1 (see [21]). If the following conditions hold.

(i) For $i \neq j, \chi_{i j}>0$.

(ii) For each $k \in \mathbb{S}$ and any $\varsigma \in R^{2}, \varrho|\varsigma|^{2} \leq \varsigma^{T} g(x, t, k) g^{T}(x, t, k) \varsigma \leq \varrho^{-1}|\varsigma|^{2}$ holds with $\varrho \in(0,1]$ for all $x \in R^{2}$.

(iii) There exists a bounded open subset $D \subset R^{2}$ with a regular boundary (i.e., smooth) such that, for any $k \in \mathbb{S}$, there exists a nonnegative function $V(\cdot, k): D^{C} \longrightarrow R$ satisfying $V(\cdot, k)$ is twice continuously differentiable and for some $\epsilon>0$,

$$
L V(x, k) \leq-\varepsilon, \quad \text { for any }(x, k) \in D^{C} \times \mathbb{S} .
$$

Then, (5) is ergodic and positive recurrent; that is, there exists a unique stationary density $\mu(\cdot, \cdot)$, for any Borel measurable function $f(\cdot, \cdot): R^{2} \times S \longrightarrow R \quad$ with $\sum_{k \in \mathbb{S}} \int_{R^{2}}|f(x, k)| \mu(x, k) \mathrm{d} x<\infty$, we have

$$
P\left(\lim _{t \longrightarrow \infty} \frac{1}{t} \int_{0}^{t} f(x(s), \alpha(s)) \mathrm{d} s=\sum_{k \in \mathbb{S}} \int_{R^{2}} f(x, k) \mu(x, k) \mathrm{d} x\right)=1 .
$$

About the existence and uniqueness of positive solutions and the moment boundedness of (2), we have the following two lemmas. The proofs of them are very standard and are omitted here. Readers may refer to $[3,21]$.

Lemma 2. There is a unique positive solution $(x(t), y(t), \alpha(t))$ for system (2) on $t \geq 0$ with initial value $(x(0), y(0), \alpha(0)) \in R_{+}^{2} \times \mathbb{S}$, and the solution will remain in $R_{+}^{2} \times \mathbb{S}$ with probability 1 .
Lemma 3. For any initial value $(x(0), y(0), \alpha(0)) \in R_{+}^{2} \times \mathbb{S}$ and any $p>0$, there exists a constant $K(p)$ such that the solution $(x(t), y(t), \alpha(t))$ for system (2) satisfying $\mathbb{E}(x(t)+y(t))^{p} \leq K(p)$ for all $t \geq 0$.

For simplicity, we give some notations as follows:

$$
\begin{aligned}
\Pi_{i}(k) & =r_{i}(k)-\frac{\sigma_{i}^{2}(k)}{2}, \quad i=1,2, \\
\Pi_{i} & =\sum_{k \in \mathbb{S}} \pi_{k} \Pi_{i}(k), \quad i=1,2, \\
\sigma & =\max _{k \in \mathbb{S}}\left\{\sigma_{1}(k), \sigma_{2}(k)\right\}, \\
\Pi(k) & =\min _{1}\left\{\Pi_{1}(k), \Pi_{2}(k)\right\}, \\
\Pi & =\sum_{k \in \mathbb{S}} \pi_{k} \Pi(k), \\
\widehat{f} & =\max _{k \in \mathbb{S}} f(k), \\
\breve{f} & =\max _{k \in \mathbb{S}} f(k) .
\end{aligned}
$$

\section{Stationary Distribution}

In this section, we discuss the stationary distribution of (2).

Theorem 1. For any initial value $(x(0), y(0), \alpha(0)) \in R_{+}^{2} \times$ $\mathbb{S}$ and any $k \in \mathbb{S}$, the solution $(x(t), y(t), \alpha(t))$ of (2) is ergodic and has a unique stationary distribution in $R_{+}^{2} \times \mathbb{S}$ if the following condition holds:

$$
\zeta=\frac{\breve{c}_{2}}{\widehat{r}_{1}+\widehat{b}_{1}}\left(\breve{r}_{1}-\frac{\widehat{\sigma}_{1}^{2}}{2}\right)-\widehat{r}_{2}-\frac{\widehat{\sigma}_{2}^{2}}{2}>0 .
$$

Proof. According to the equivalent property of (2) and (4), we only need to prove it for (4). Define $V_{1}(t, u, v)=\left(\left(e^{u}+p e^{v}\right)^{2} / 2\right)$, where $p=\left(\widetilde{c}_{1} / \widehat{c}_{2}\right)$, and then we have 


$$
\begin{aligned}
& L V_{1}=\left(e^{u}+p e^{v}\right) e^{u}\left(r_{1}(\alpha(t))-\frac{\sigma_{1}^{2}(\alpha(t))}{2}-b_{1}(\alpha(t)) e^{u}-\frac{c_{1}(\alpha(t)) e^{v}}{1+e^{u}}\right) \\
& +p\left(e^{u}+p e^{v}\right) e^{v}\left(-r_{2}(\alpha(t))-\frac{\sigma_{2}^{2}(\alpha(t))}{2}-b_{2}(\alpha(t)) e^{v}+\frac{c_{2}(\alpha(t)) e^{v}}{1+e^{u}}\right) \\
& +\frac{\sigma_{1}^{2}(\alpha(t)}{2}\left(e^{2 u}+e^{u}\left(e^{u}+p e^{v}\right)\right)+\frac{\sigma_{2}^{2}(\alpha(t)}{2}\left(p^{2} e^{2 v}+p e^{v}\left(e^{u}+p e^{v}\right)\right) \\
& =\left(e^{u}+p e^{v}\right)\left[e^{u}\left(r_{1}(\alpha(t))-\frac{\sigma_{1}^{2}(\alpha(t))}{2}\right)-b_{1}(\alpha(t)) e^{2 u}-\frac{c_{1}(\alpha(t)) e^{u+v}}{1+e^{u}}\right] \\
& +\left(e^{u}+p e^{v}\right)\left[-p e^{v}\left(r_{2}(\alpha(t))+\frac{\sigma_{2}^{2}(\alpha(t))}{2}\right)-p b_{2}(\alpha(t)) e^{2 v}+\frac{p c_{2}(\alpha(t)) e^{u+v}}{1+e^{u}}\right] \\
& +\left(e^{u}+p e^{v}\right)\left(\frac{\sigma_{1}^{2}(\alpha(t))}{2} e^{u}+\frac{\sigma_{2}^{2}(\alpha(t))}{2} p e^{v}\right)+\frac{\sigma_{1}^{2}(\alpha(t))}{2} e^{2 u}+\frac{\sigma_{2}^{2}(\alpha(t))}{2} p^{2} e^{2 v} \\
& \leq\left(e^{u}+p e^{v}\right)\left[e^{u}\left(r_{1}(\alpha(t))-\frac{\sigma_{1}^{2}(\alpha(t))}{2}\right)-\breve{b}_{1} e^{2 u}-p e^{v}\left(r_{2}(\alpha(t))+\frac{\sigma_{2}^{2}(\alpha(t))}{2}\right)\right. \\
& \left.-p \breve{b}_{2} e^{2 v}\right]+\frac{\left(e^{u}+p e^{v}\right)}{2}\left(\sigma_{1}^{2}(\alpha(t)) e^{u}+p \sigma_{2}^{2}(\alpha(t)) e^{v}\right)+\frac{\sigma_{1}^{2}(\alpha(t))}{2} e^{2 u}+\frac{\sigma_{2}^{2}(\alpha(t))}{2} p^{2} e^{2 v} \leq \\
& \leq\left(r_{1}(k)-\frac{\sigma_{1}^{2}(k)}{2}\right)\left(e^{u}+p e^{v}\right) e^{u}-\breve{b}_{1} e^{3 u}-p^{2} \breve{b}_{2} e^{3 v}+\sigma_{1}^{2}(\alpha(t)) e^{2 u}+p^{2} \sigma_{2}^{2}(\alpha(t)) e^{2 v} \\
& +\frac{p\left(\sigma_{1}^{2}(k)+\sigma_{2}^{2}(k)\right)}{2} e^{u+v} \leq \\
& -\frac{\breve{b}_{1}}{2} e^{3 u}-\frac{p^{2} \breve{b}_{2}}{2} e^{3 v}+\varrho
\end{aligned}
$$

where

$$
\begin{aligned}
\varrho= & \sup _{u, v \in R^{2}}\left\{-\frac{\breve{b}_{1}}{2} e^{3 u}-\frac{p^{2} \breve{b}_{2}}{2} e^{3 v}+\left(r_{1}(k)-\frac{\sigma_{1}^{2}(k)}{2}\right)\left(e^{u}+p e^{v}\right) e^{u}\right. \\
& \left.+\sigma_{1}^{2}(k) e^{2 u}+p^{2} \sigma_{2}^{2}(k) e^{2 v}+\frac{p\left(\sigma_{1}^{2}(k)+\sigma_{2}^{2}(k)\right)}{2} e^{u+v}\right\} .
\end{aligned}
$$

On the other hand,

$$
L(-v)=r_{2}(\alpha(t))+\frac{\sigma_{2}^{2}(\alpha(t))}{2}+b_{2}(\alpha(t)) e^{v}-\frac{c_{2}(\alpha(t)) e^{u}}{1+e^{u}}
$$

$$
\begin{gathered}
\leq r_{2}(\alpha(t))+\frac{\sigma_{2}^{2}(\alpha(t))}{2}+\widehat{b}_{2} e^{v}-\frac{\breve{c}_{2} e^{u}}{1+e^{u}} \\
\leq r_{2}(k)+\frac{\sigma_{2}^{2}(k)}{2}+\widehat{b}_{2} e^{v}-\frac{\breve{c}_{2} r_{1}(k)}{r_{1}(k)+b_{1}(k)} \\
+\frac{\breve{c}_{2}}{r_{1}(k)+b_{1}(k)} \frac{r_{1}(k)-b_{1}(k) e^{u}}{1+e^{u}} .
\end{gathered}
$$

Set $\widetilde{q}=\left(\left(\widehat{c_{1}}+\widehat{b_{2}}\right) / \check{r_{2}}\right)$, and similarly we have 


$$
\begin{aligned}
& L\left(\ln \left(1+e^{u}\right)-u+\widetilde{q} e^{v}\right)=\frac{e^{u}}{1+e^{u}}\left[r_{1}(\alpha(t))-\frac{\sigma_{1}^{2}(\alpha(t))}{2}-b_{1}(\alpha(t)) e^{u}-\frac{c_{1}(\alpha(t)) e^{v}}{1+e^{u}}\right]+\frac{\sigma_{1}^{2}(\alpha(t))}{2} \\
& \times \frac{e^{u}}{\left(1+e^{u}\right)^{2}}+\left[-r_{1}(\alpha(t))+\frac{\sigma_{1}^{2}(\alpha(t))}{2}+b_{1}(\alpha(t)) e^{u}+\frac{c_{1}(\alpha(t)) e^{v}}{1+e^{u}}\right] \\
& +\widetilde{q}\left[\left(e^{v}\left(-r_{2}(\alpha(t))\right)-\frac{\sigma_{2}^{2}(\alpha(t))}{2}-b_{2}(\alpha(t)) e^{v}+\frac{c_{2}(\alpha(t)) e^{u}}{1+e^{u}}\right)+\frac{\sigma_{2}^{2}(\alpha(t))}{2} e^{v}\right] \\
& =\left[\frac{e^{u} r_{1}\left((\alpha(t))-b_{1}(\alpha(t)) e^{u}\right)}{1+e^{u}}-\frac{c_{1}(\alpha(t)) e^{u+v}}{\left(1+e^{u}\right)^{2}}-\frac{\left.\sigma_{1}^{2}(\alpha(t)) e^{2 u}\right)}{2\left(1+e^{u}\right)^{2}}\right] \\
& +\left[-r_{1}(\alpha(t))+\frac{\sigma_{1}^{2}(\alpha(t))}{2}+b_{1}(\alpha(t)) e^{u}+\frac{c_{1}(\alpha(t)) e^{v}}{1+e^{u}}\right] \\
& \left.+\widetilde{q}\left[-r_{2}(\alpha(t)) e^{v}-b_{2}(\alpha(t)) e^{2 v}+\frac{c_{2}(\alpha(t)) e^{u+v}}{1+e^{u}}\right)\right] \\
& \leq\left[\left(r_{1}(k)-b_{1}(k)\right) e^{u}-\frac{r_{1}(k)-b_{1}(k) e^{u}}{1+e^{u}}\right]-r_{1}(k)+\frac{\sigma_{1}^{2}(k)}{2}+b_{1}(k) e^{u}+c_{1}(k) e^{v} \\
& +\widetilde{q}\left[-r_{2}(\alpha(t)) e^{v}+c_{2}(k) e^{u+v}\right] \text {. }
\end{aligned}
$$

Define $V_{2}=-v+\left(\breve{c}_{2} / r_{1}(\alpha(t))+b_{1}(\alpha(t))\right)\left(\ln \left(1+e^{u}\right)-\right.$ $\left.u+\widetilde{q} e^{v}\right)$, and then

$$
\begin{aligned}
L V_{2} \leq & r_{2}(\alpha(t))+\frac{\sigma_{2}^{2}(\alpha(t))}{2}+\widehat{b}_{2} e^{v}-\frac{\breve{c}_{2} r_{1}(\alpha(t))}{r_{1}(\alpha(t))+b_{1}(\alpha(t))}+\frac{\breve{c}_{2}}{r_{1}(\alpha(t))+b_{1}(\alpha(t))} \frac{r_{1}(\alpha(t))-b_{1}(\alpha(t)) e^{u}}{1+e^{u}} \\
& +\frac{\breve{c}_{2}}{r_{1}(\alpha(t))+b_{1}(\alpha(t))}\left\{\left[\left(r_{1}(\alpha(t))-b_{1}(\alpha(t))\right) e^{u}-\frac{r_{1}(\alpha(t))-b_{1}(\alpha(t)) e^{u}}{1+e^{u}}\right]-r_{1}(\alpha(t))\right. \\
& \left.+\frac{\sigma_{1}^{2}(\alpha(t))}{2}+b_{1}(\alpha(t)) e^{u}+c_{1}(\alpha(t)) e^{v}+\widetilde{q}\left[-r_{2}(\alpha(t)) e^{v}+c_{2}(\alpha(t)) e^{u+v}\right]\right\} \\
\leq & r_{2}(k)+\frac{\sigma_{2}^{2} \cdot(k)}{2}-\frac{\breve{c}_{2} r_{1}(k)}{r_{1}(k)+b_{1}(k)}+\frac{\breve{c}_{2}}{r_{1}(k)+b_{1}(k)}\left[\frac{\sigma_{1}^{2}(k)}{2}+\widetilde{q}_{\widehat{c}} e^{u+v}\right] \\
\leq & -\frac{\breve{c}_{2}}{r_{1}(k)+b_{1}(k)}\left(r_{1}(k)-\frac{\sigma_{1}^{2}(k)}{2}\right)+r_{2}(k)+\frac{\sigma_{2}^{2}(k)}{2}+\frac{\breve{c}_{2}}{\widehat{r}_{1}+\widehat{b}_{1}} \widetilde{q}_{2} e^{u+v} \\
\leq & -\zeta+q e^{u+v},
\end{aligned}
$$

where $q=\left(\breve{c}_{2} \widehat{c}_{2} / \widehat{r}_{1}+\widehat{b}_{1}\right) \widetilde{q}$. Let $V=V_{1}+M V_{2}$, where $M=$ $(2 / \zeta) \max \left\{2, \sup _{(u, v) \in R_{+}^{2}}\left(-\left(b_{1} / 4\right) e^{3 u}-\left(p^{2} b_{2} / 4\right) e^{3 v}+\varrho\right)\right\}$. It is easy to observe that

$$
L V=L V_{1}+M L V_{2} \leq-M \zeta+M q e^{u+v}-\frac{\breve{b}_{1}}{2} e^{3 u}-\frac{p^{2} \breve{b}_{2}}{2} e^{3 v}+\varrho,
$$

and

$$
\frac{M \zeta}{4} \geq 1
$$

Define a bounded closed set as follows:

$$
U=\left\{(u, v):|u| \leq \ln \varepsilon^{-1},|v| \leq \ln \varepsilon^{-1},(u, v) \in R^{2}\right\},
$$


where $\varepsilon$ is a sufficiently small number, and then the set $U^{C}=$ $\left(R_{+}^{2} / U\right)$ contains the following four domains:

$$
\begin{aligned}
U_{\varepsilon}^{1} & =\left\{(u, v) \in R^{2}:-\infty \leq u \leq \ln \varepsilon\right\}, \\
U_{\varepsilon}^{2} & =\left\{(u, v) \in R^{2}:-\infty \leq v \leq \ln \varepsilon\right\}, \\
U_{\varepsilon}^{3} & =\left\{(u, v) \in R^{2}: u \geq \ln \varepsilon^{-1}\right\}, \\
U_{\varepsilon}^{4} & =\left\{(u, v) \in R^{2}: v \geq \ln \varepsilon^{-1}\right\} .
\end{aligned}
$$

Take $\varepsilon$ sufficiently small enough such that

$$
\begin{aligned}
& 0<\varepsilon<\frac{\zeta}{4 q}, \\
& 0<\varepsilon<\frac{p^{2} \breve{b}_{2}}{4 M q}, \\
& 0<\varepsilon<\frac{\breve{b_{1}}}{4 M q},
\end{aligned}
$$

$$
\begin{aligned}
& -M \zeta-\frac{\breve{b_{1}}}{4 \varepsilon}+\eta_{1} \leq-1, \\
& -\zeta-\frac{p^{2} \breve{b}_{2}}{4 \varepsilon}+\eta_{2} \leq-1,
\end{aligned}
$$

where $\eta_{1}, \eta_{2}$ are defined later. Next, we verify $L V(u, v) \leq-1$ for all $(u, v) \in U^{C}=U_{\varepsilon}^{1} \cup U_{\varepsilon}^{2} \cup U_{\varepsilon}^{3} \cup U_{\varepsilon}^{4}$.

Case 1. If $(u, v) \in U_{\varepsilon}^{1}$, namely, $-\infty \leq u \leq \ln \varepsilon$, then $e^{u+v} \leq \varepsilon e^{v} \leq \varepsilon\left(1+e^{3 v}\right)$. By (17), (18), and (21), we have

$$
\begin{aligned}
L V \leq & -M \zeta+M q \varepsilon e^{v}-\frac{\breve{b}_{1}}{2} e^{3 u}-\frac{p^{2} \breve{b}_{2}}{2} e^{3 v}+\varrho \\
\leq & -M \zeta+M q \varepsilon+M q \varepsilon e^{3 v}-\frac{\breve{b}_{1}}{2} e^{3 u}-\frac{p^{2} \breve{b}_{2}}{2} e^{3 v}+\varrho \\
& =\frac{-M \zeta}{4}+\left(\frac{-M \zeta}{4}+M q \varepsilon\right)+\left(-\frac{p^{2} \breve{b}_{2}}{4}+M q \varepsilon\right) e^{3 v}-\frac{\breve{b}_{1}}{4} e^{3 u}+\left(\frac{-M \zeta}{2}-\frac{p^{2} \breve{b}_{2}}{4} e^{3 v}-\frac{\breve{b}_{1}}{4} e^{3 u}+\varrho\right) \\
\leq & \left.\frac{-M \zeta}{4}+\left(\frac{-M \zeta}{4}+M q \varepsilon\right)+\left(-\frac{p^{2} \breve{b}}{4}+M q \varepsilon\right) e^{3 v}-\frac{\breve{b}_{1}}{4} e^{3 u}\right) \\
& +\left[\frac{-M \zeta}{2}+\sup _{(u, v) \in R_{+}^{2}}\left(-\frac{p^{2} \breve{b}_{2}}{4} e^{3 v}-\frac{\breve{b}_{1}}{4} e^{3 u}+\varrho\right)\right] \\
\leq & -1 .
\end{aligned}
$$


Case 2. If $(u, v) \in U_{\varepsilon}^{2}$, namely, $-\infty \leq v \leq \ln \varepsilon$, then $e^{u+v} \leq \varepsilon e^{u} \leq \varepsilon\left(1+e^{3 u}\right)$, and similarly we have

$$
\begin{aligned}
L V \leq & -M \zeta+M q \varepsilon e^{u}-\frac{\breve{b}_{1}}{2} e^{3 u}-\frac{p^{2} \breve{b}_{2}}{2} e^{3 v}+\varrho \\
\leq & \frac{-M \zeta}{4}+\left(\frac{-M \zeta}{4}+M q \varepsilon\right)+\left(-\frac{\breve{b}_{1}}{4}+M q \varepsilon\right) e^{3 u}-\frac{p^{2} \breve{b}_{2}}{4} e^{3 v} \\
& +\left[\frac{-M \zeta}{2}+\sup _{(u, v) \in R_{+}^{2}}\left(-\frac{\breve{b}_{1}}{4} e^{3 u}-\frac{p^{2} \breve{b}_{2}}{4} e^{3 v}+\varrho\right)\right] \\
\leq & -1 .
\end{aligned}
$$

Case 3. If $(u, v) \in U_{\varepsilon}^{3}$, then we derive from (17) and (22) that

$$
\begin{aligned}
L V & \leq-M \zeta+M q \varepsilon e^{u}-\frac{\breve{b}_{1}}{2} e^{3 u}-\frac{p^{2} \breve{b}_{2}}{2} e^{3 v}+\varrho \\
& \leq-M \zeta-\frac{\breve{b}_{1}}{4 \varepsilon}+\eta_{1} \\
& \leq-1,
\end{aligned}
$$

where

$\eta_{1}=\sup _{(u, v) \in R_{+}^{2}}\left(-\left(\breve{b}_{1} / 4\right) e^{3 u}-\left(p^{2} \breve{b}_{2} / 2\right) e^{3 v}+M q e^{u+v}+\varrho\right)$.

Case 4. If $(u, v) \in U_{\mathcal{\varepsilon}}^{4}$, similarly, from (17) and (22) we have

$$
\begin{aligned}
L V & \leq-M \zeta+M q \varepsilon e^{u}-\frac{\breve{b}_{1}}{2} e^{3 u}-\frac{p^{2} \breve{b_{2}}}{2} e^{3 v}+\varrho \\
& \leq-M \zeta-\frac{p^{2} \breve{b}_{2}}{4 \varepsilon}+\eta_{2} \\
& \leq-1,
\end{aligned}
$$

where

$\eta_{2}=\sup _{(u, v) \in R_{+}^{2}}\left(-\left(\breve{b}_{1} / 2\right) e^{3 u}-\left(p^{2} \breve{b}_{2} / 4\right) e^{3 v}+M q e^{u+v}+\varrho\right)$.
Consequently, we deduce that $L V(u, v) \leq-1$ on all $(u, v) \in U^{C}$. Obviously, the other condition of Lemma 1 holds too, so we conclude from Lemma 1 that system (4) is ergodic and has a unique stationary distribution in $R_{+}^{2} \times \mathbb{S}$; that is, system (2) is ergodic and has a unique stationary distribution in $R_{+}^{2} \times \mathbb{S}$. This completes the proof.

For (2), if the state Markovian chain $\alpha(t)$ takes value in space $\mathbb{S}=\{1\}$, namely, there is no switching, then (2) turns to the following subsystem:

$$
\left\{\begin{array}{l}
\mathrm{d} x(t)=x(t)\left(r_{1}-b_{1} x(t)-\frac{c_{1} y(t)}{1+x(t)}\right) \mathrm{d} t+\sigma_{1} x(t) \mathrm{d} B_{1}(t), \\
\mathrm{d} y(t)=y(t)\left(-r_{2}-b_{2} y(t)+\frac{c_{2} x(t)}{1+x(t)}\right) \mathrm{d} t+\sigma_{2} y(t) \mathrm{d} B_{2}(t) .
\end{array}\right.
$$

For (27), from Theorem 1, we can easily obtain the following conclusion.

Corollary 1. For any initial value $(x(0), y(0)) \in R_{+}^{2}$, the solution $(x(t), y(t))$ of (27) is ergodic and has a unique stationary distribution in $R_{+}^{2}$ if the following condition holds:

$$
\tilde{\zeta}=\frac{c_{2}}{r_{1}+b_{1}}\left(r_{1}-\frac{\sigma_{1}^{2}}{2}\right)-r_{2}-\frac{\sigma_{2}^{2}}{2}>0 .
$$

Remark 1. It is clear that, for any positive integer $k,\left(c_{2}(k) / r_{1}(k)+b_{1}(k)\right)\left(r_{1}(k)-\left(\sigma_{1}^{2}(k) / 2\right)\right)-r_{2}(k)-\left(\sigma_{2}^{2}(k)\right.$ /2) $>\left(\breve{c}_{2} / \widehat{r}_{1}+\widehat{b}_{1}\right)\left(\breve{r}_{1}-\left(\widehat{\sigma}_{1}^{2} / 2\right)\right)-\widehat{r}_{2}-\left(\widehat{\sigma}_{2}^{2} / 2\right)$. That is, Theorem 1 shows that switching system (2) has stationary distribution only under the condition that every subsystem of (2) has stationary distribution. If there exists no switching, Corollary 1 gives the sufficient condition of stationary distribution of (27), which is accordant with Theorem 1 of [3].

\section{Stochastic Permanence}

For (2), if we consider the birth rate instead of the death rate of predator, then (2) turns to the following model:

$$
\left\{\begin{array}{l}
\mathrm{d} x(t)=x(t)\left(r_{1}(\alpha(t))-b_{1}(\alpha(t)) x(t)-\frac{c_{1}(\alpha(t)) y(t)}{1+x(t)}\right) \mathrm{d} t+\sigma_{1}(\alpha(t)) x(t) \mathrm{d} B_{1}(t) \\
\mathrm{d} y(t)=y(t)\left(r_{2}(\alpha(t))-b_{2}(\alpha(t)) y(t)+\frac{c_{2}(\alpha(t)) x(t)}{1+x(t)}\right) \mathrm{d} t+\sigma_{2}(\alpha(t)) y(t) \mathrm{d} B_{2}(t)
\end{array}\right.
$$

where $r_{2}(\cdot)>0$ is the birth rate of species $y(t)$ and other parameters are the same as before. Now, we consider the stochastic permanence of (29).
Definition 1. (see [16])System (29) is stochastically permanent if for every $\varepsilon \in(0,1)$ and any $k \in \mathbb{S}$, there is a pair of constants $\mathscr{M}>0$ and $\mathscr{N}>0$ such that for any initial data 
$(x(0), y(0), \alpha(0)) \in R_{+}^{2} \times \mathbb{S}, \quad$ the solution $X(t)=(x(t), y(t))$ of $(29)$ has the property that

$$
\begin{gathered}
\liminf _{t \longrightarrow \infty} P\{|X(t)| \geq \mathscr{M}\} \geq 1-\varepsilon, \\
\limsup _{t \rightarrow \infty} P\{|X(t)| \leq \mathscr{N}\} \geq 1-\varepsilon,
\end{gathered}
$$

where $P$ represents the probability of events.

Assumption 1. For some $k \in \mathbb{S}, \chi_{k j}>0, k \neq j$.

Lemma 4. Under Assumption 1, if $\Pi>0$, then there exists $\delta_{0}>0$ such that for any $0<\delta<\delta_{0}, G(\delta)=\operatorname{diag}\left(v_{1}(\delta), v_{2}(\delta), \ldots, v_{N}(\delta)\right)-\chi \quad$ is $a$ nonsingular M-matrix, where $v_{k}(\delta)=\delta \Pi(k)-(1 / 2) \delta^{2} \sigma^{2}$.

Remark 2. The proof is rather standard. Readers may refer to the details in [24] or [21, 23].
Theorem 2. For any initial value $(x(0), y(0), \alpha(0)) \in R_{+}^{2} \times \mathbb{S}$, system (29) is stochastically permanent under conditions of Lemma 3.

Proof. The proof is motivated by [22]. Let $G$ be a matrix or vector, and denote by $G \gg 0$ all the elements of $G$ are positive. Under the hypotheses, Lemma 2 shows $G(\delta)$ is a nonsingular M-matrix, and then by M-matrix theory (see Theorem 2.1 [22]), there exists $\rho=\left(\rho_{1}, \rho_{2}, \ldots, \rho_{N}\right)^{T} \gg 0$ such that $G(\delta) \rho \gg 0$, that is, $\rho_{k} v_{k}(\delta)-\sum_{j=1}^{N} \chi_{k j} \rho_{j}>0, k \in \mathbb{S}$. So, there exists a constant $\tau>0$ such that

$$
\rho_{k} v_{k}(\delta)-\sum_{j=1}^{N} \chi_{k j} \rho_{j}-\tau \rho_{k}>0, \quad k \in \mathbb{S} .
$$

Define functional $\quad V=\rho_{k}(1+\tilde{V})^{\delta}, \quad$ where $\widetilde{V}=(1 /(x+y))$, then for above $\tau>0$, we compute $\tau V+L V$ as follows:

$$
\begin{aligned}
& \tau V+L V=\tau \rho_{k}(1+\widetilde{V})^{\delta}+\sum_{j=1}^{N} \chi_{k j} \rho_{j}(1+\widetilde{V})^{\delta}+\rho_{k} \delta(1+\widetilde{V})^{\delta-1}\left(-\tilde{V}^{2}\right) \\
& \times\left(r_{1}(\alpha(t)) x+r_{2}(\alpha(t)) y-b_{1}(\alpha(t)) x^{2}-b_{2}(\alpha(t)) y^{2}+\frac{c_{2}(\alpha(t))-c_{1}(\alpha(t))}{1+x} x y\right) \\
& +\rho_{k} \delta(1+\widetilde{V})^{\delta-1} \widetilde{V}\left(\frac{\sigma_{1}(\alpha(t)) x+\sigma_{2}(\alpha(t)) y}{x+y}\right)^{2} \\
& +\frac{1}{2} \rho_{k} \delta(\delta-1)(1+\widetilde{V})^{\delta-2}\left[-\widetilde{V}^{2}\left(\sigma_{1}(\alpha(t))\right) x-\sigma_{2}((\alpha(t)) y)^{2}\right] \\
& =\tau \rho_{k}(1+\widetilde{V})^{\delta}+\sum_{j=1}^{N} \chi_{k j} \rho_{j}(1+\widetilde{V})^{\delta}-\rho_{k} \delta(1+\widetilde{V})^{\delta-1} \widetilde{V} \frac{r_{1}(\alpha(t)) x+r_{2}(\alpha(t)) y}{x+y} \\
& +\rho_{k} \delta(1+\tilde{V})^{\delta-1} \frac{b_{1}(\alpha(t)) x^{2}+b_{2}(\alpha(t)) y^{2}-\left(\left(c_{2}(\alpha(t))-c_{1}(\alpha(t))\right) /(1+x)\right) x y}{(x+y)^{2}} \\
& +\rho_{k} \delta(1+\widetilde{V})^{\delta-1} \widetilde{V}\left(\frac{\sigma_{1}(\alpha(t)) x+\sigma_{2}(\alpha(t)) y}{x+y}\right)^{2} \\
& +\frac{1}{2} \rho_{k} \delta(\delta-1)(1+\widetilde{V})^{\delta-2} \widetilde{V}^{2}\left(\frac{\sigma_{1}(\alpha(t)) x+\sigma_{2}(\alpha(t)) y}{x+y}\right)^{2} \\
& =\widetilde{V}^{\delta}\left(f\left(\widetilde{V}^{\delta}\right)\right)+g(\widetilde{V}),
\end{aligned}
$$


where $\lim _{V \longrightarrow \infty}\left(g(\widetilde{V}) / \widetilde{V}^{\delta}\right)=0$, and

$$
\begin{aligned}
f\left(\widetilde{V}^{\delta}\right)= & \tau \rho_{k}+\sum_{j=1}^{N} \chi_{k j} \rho_{j}-\rho_{k} \delta \frac{r_{1}(\alpha(t)) x+r_{2}(\alpha(t)) y}{x+y}+\rho_{k} \delta\left(\frac{\sigma_{1}(\alpha(t)) x+\sigma_{2}(\alpha(t)) y}{x+y}\right)^{2} \\
& +\frac{1}{2} \rho_{k} \delta(\delta-1)\left(\frac{\sigma_{1}(\alpha(t)) x+\sigma_{2}(\alpha(t)) y}{x+y}\right)^{2} \\
\leq & \tau \rho_{k}+\sum_{j=1}^{N} \chi_{k j} \rho_{j}-\rho_{k} \delta \frac{\Pi_{1}(k) x}{x+y}-\rho_{k} \delta \frac{\Pi_{2}(k) y}{x+y}+\frac{\rho_{k} \delta^{2} \sigma^{2}}{2} \\
\leq & \tau \rho_{k}+\sum_{j=1}^{N} \chi_{k j} \rho_{j}-\rho_{k} \Pi(k) \delta+\frac{\rho_{k} \delta^{2} \sigma^{2}}{2} \\
= & \tau \rho_{k}+\sum_{j=1}^{N} \chi_{k j} \rho_{j}-\rho_{k} v_{k}(\delta)
\end{aligned}
$$

$<0$.

By Ito's formula, we have

$$
\begin{aligned}
L\left(e^{\tau t} V(t)\right) & =e^{\tau t}(\tau V+L V) \\
& \leq e^{\tau t}\left(\left(\tau \rho_{k}-\rho_{k} v_{k}(\delta)+\sum_{j=1}^{N} \chi_{k j} \rho_{j}\right) \widetilde{V}^{\delta}+o\left(\widetilde{V}^{\delta}\right)\right) \\
& \leq Y(\delta) e^{\tau t},
\end{aligned}
$$

where $Y(\delta)=\left(\max _{k \in \mathbb{S}} \sup _{\widetilde{V} \in R^{2}}\left(\left(\tau \rho_{k}-\rho_{k} v_{k}(\delta)+\sum_{j=1}^{N} \chi_{k j} \rho_{j}\right)\right.\right.$ $\left.\widetilde{V}^{\delta}+o\left(\widetilde{V}^{\delta}\right)\right)$, 1). Integrating $d\left(e^{\tau t} V(t)\right)$ from 0 to $t$ and taking expectation give

$$
\mathbb{E}\left[\rho_{k} e^{\tau t}(1+\widetilde{V})^{\delta}\right]-\rho_{k}(1+\widetilde{V}(0))^{\delta} \leq \frac{Y(\delta)}{k}\left(e^{\tau t}-1\right) .
$$

Hence,

$\mathbb{E}(1+\widetilde{V})^{\delta} \leq \frac{Y(\delta)}{k \min _{k \in \mathbb{S}} \rho_{k}}+e^{-\tau t}\left(1+\frac{1}{x(0)+y(0)}\right)^{\delta}$.

Let $H(\delta)=\left(Y(\delta) / k \min _{k \in \mathbb{S}} \rho_{k}\right)$, then

$$
\limsup _{t \longrightarrow \infty} \mathbb{E}(x(t)+y(t))^{-\delta} \leq H(\delta) .
$$

Since $|X|=\left(x^{2}+y^{2}\right)^{(1 / 2)}$, then we deduce that $\mathbb{E}|X|^{-\delta} \leq 2^{(\delta / 2)} H(\delta)$, and hence,

$$
P\left\{|X|<\frac{\sqrt{2}}{2}\left(\frac{\varepsilon}{H(\delta)}\right)^{(1 / \delta)}=\mathscr{M}\right\} \leq \frac{\mathbb{E}|X|^{-\delta}}{\mathscr{M}^{-\delta}} \leq \frac{2^{(\delta / 2)} H(\delta)}{2^{(\delta / 2)}(\varepsilon / H(\delta))^{-1}}=\varepsilon .
$$

Therefore, $P\{|X| \geq \mathscr{M}\} \geq 1-\varepsilon$ holds. By Lemma 3, using Chebyshev's inequality again, it is clear that $P\{|X| \leq \mathscr{N}\} \geq 1-$ $\varepsilon$ for some constant $\mathcal{N}$. Therefore, (29) is stochastically permanent by Definition 1. The proof is completed.

Obviously, if there is no switching, we can similarly obtain the following corollary.

Corollary 2. For any initial value $(x(0), y(0)) \in R_{+}^{2}$, the subsystem of (29) is stochastically permanent if $r_{i}-\left(\sigma_{i}^{2} / 2\right)>0, i=1,2$.

Remark 3. Theorem 2 reveals that when some subsystems of (2) are no stochastic permanent, if we give a suitable switching, then switching system (2) may be stochastic permanent, which implies the switching has very important influence to the dynamics of (2). By simulation, we can verify it directly, see Figure 1.

\section{Examples and Simulations}

In this section, some examples are given to illustrate our theoretical results and reveal the effects of regime switching and stochastic factors [25]. For simplicity, we assume that the continuous-time discrete state Markovian chain $\alpha(t)$ takes value in the space $\mathbb{S}=\{1,2\}$, then system (2) reduces to the following subsystems: 


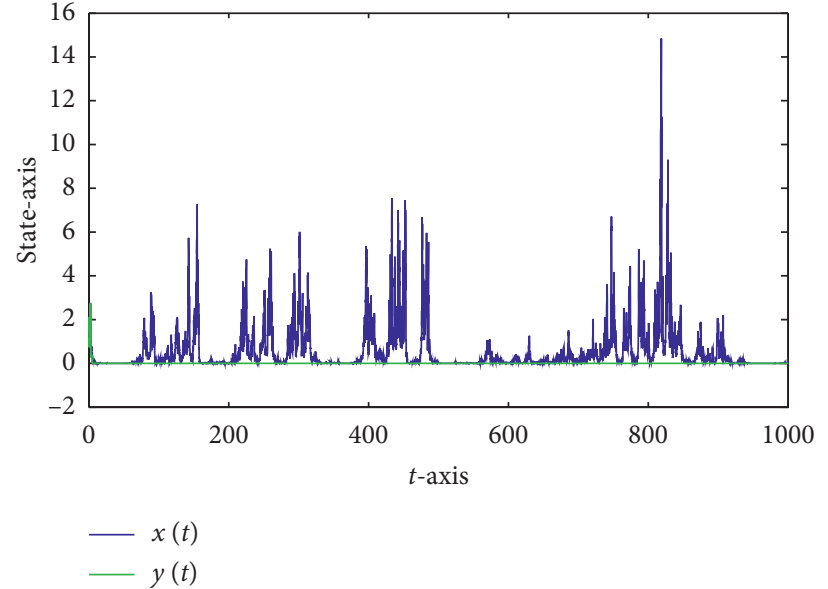

(a)

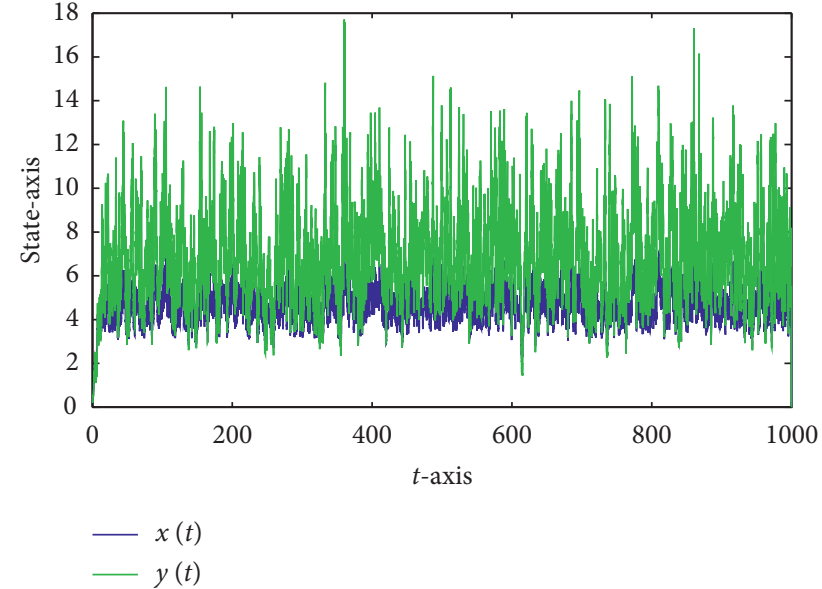

(b)

FIGURE 1: The effect of switching $\pi$ on the dynamics of (39) and (40). (a) The nonstochastic permanence of $(39)$ and $(40)$ with $\pi=(0.1,0.9)$. (b) The stochastic permanence of (39) and (40) with $\pi=(0.1,0.9)$.
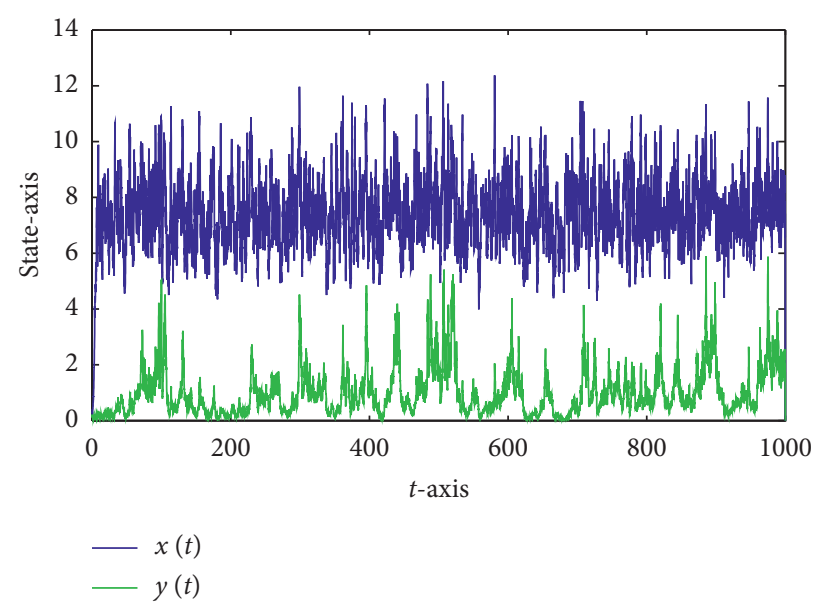

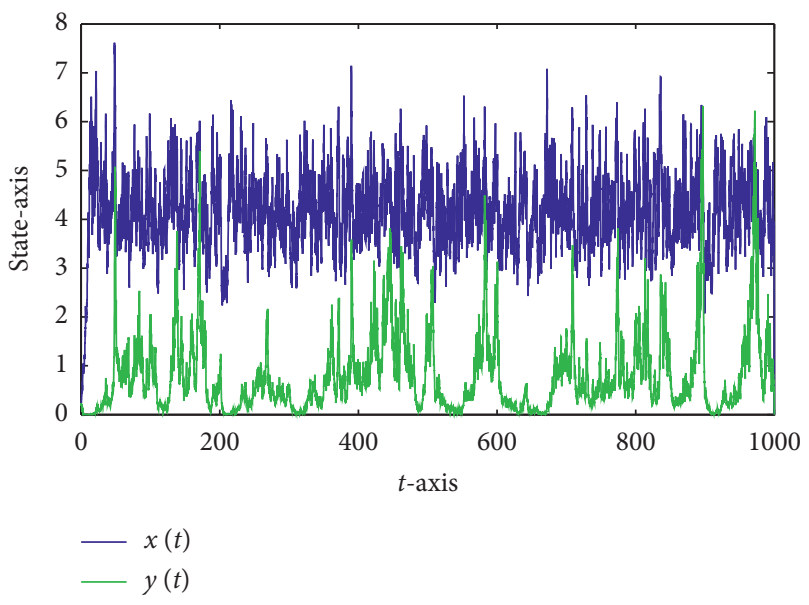

(b)

Figure 2: (a) The stationary distribution of system (39). (b) The stationary distribution of system (40).

$$
\begin{aligned}
& \left\{\begin{array}{l}
\mathrm{d} x(t)=x(t)\left(r_{1}(1)-b_{1}(1) x(t)-\frac{c_{1}(1) y(t)}{1+x(t)}\right) \mathrm{d} t+\sigma_{1}(1) x(t) \mathrm{d} B_{1}(t), \\
\mathrm{d} y(t)=y(t)\left(-r_{2}(1)-b_{2}(1) y(t)+\frac{c_{2}(1) x(t)}{1+x(t)}\right) \mathrm{d} t+\sigma_{2}(1) y(t) \mathrm{d} B_{2}(t),
\end{array}\right. \\
& \left\{\begin{array}{l}
\mathrm{d} x(t)=x(t)\left(r_{1}(2)-b_{1}(2) x(t)-\frac{c_{1}(2) y(t)}{1+x(t)}\right) \mathrm{d} t+\sigma_{1}(2) x(t) \mathrm{d} B_{1}(t), \\
\mathrm{d} y(t)=y(t)\left(-r_{2}(2)-b_{2}(2) y(t)+\frac{c_{2}(2) x(t)}{1+x(t)}\right) \mathrm{d} t+\sigma_{2}(2) y(t) \mathrm{d} B_{2}(t) .
\end{array}\right.
\end{aligned}
$$




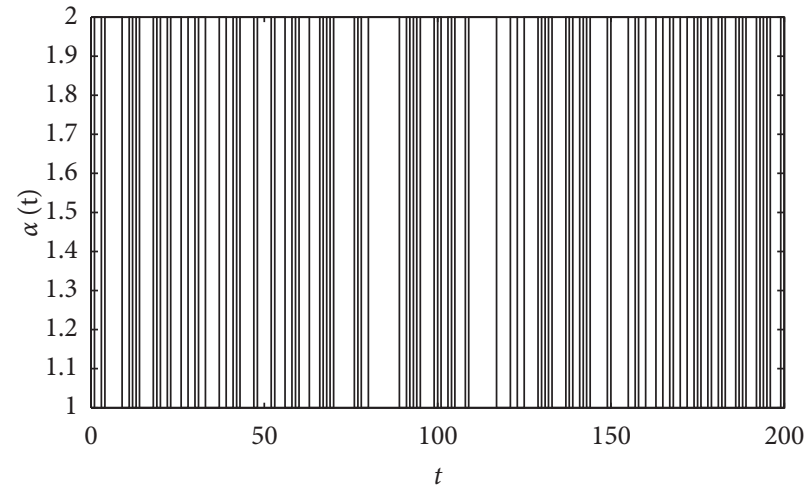

(a)

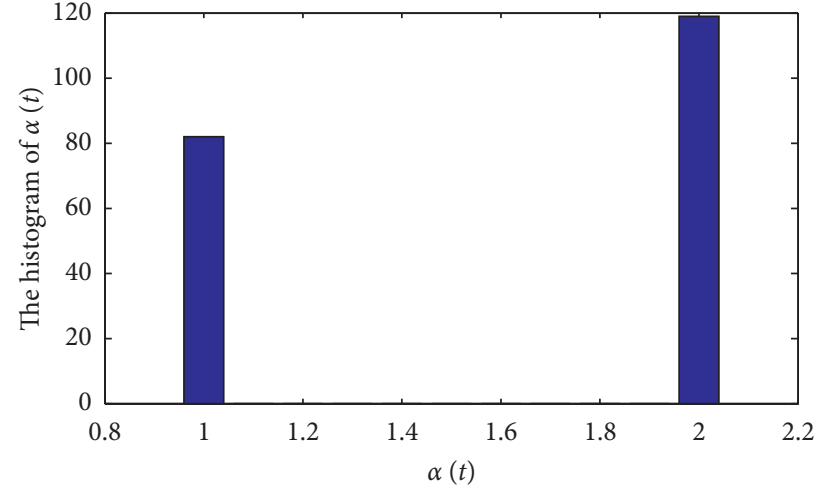

(b)

Figure 3: Distribution of the switching $\alpha(t)$. (a) The time series of $\alpha(t)$. (b) The histogram of $\alpha(t)$.

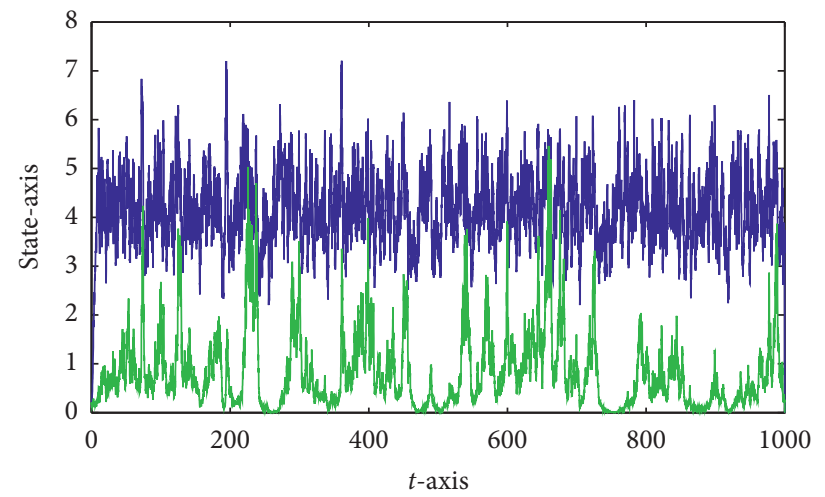

$x(t)$
$-y(t)$

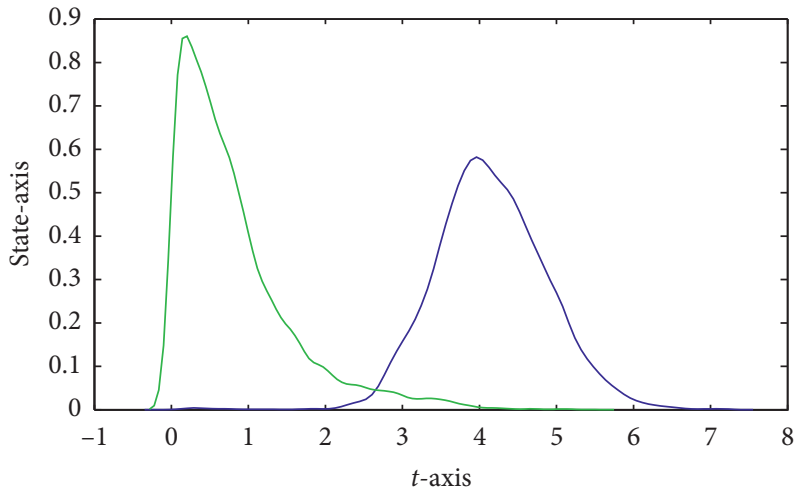

$-x(t)$

(a)

(b)

Figure 4: The stationary distribution of switching systems (39) and (40) with $\pi=(0.4,0.6)$. (a) The time series graph of $(x(t)$ and $y(t))$. (b) The density graph of $(x(t)$ and $y(t))$.

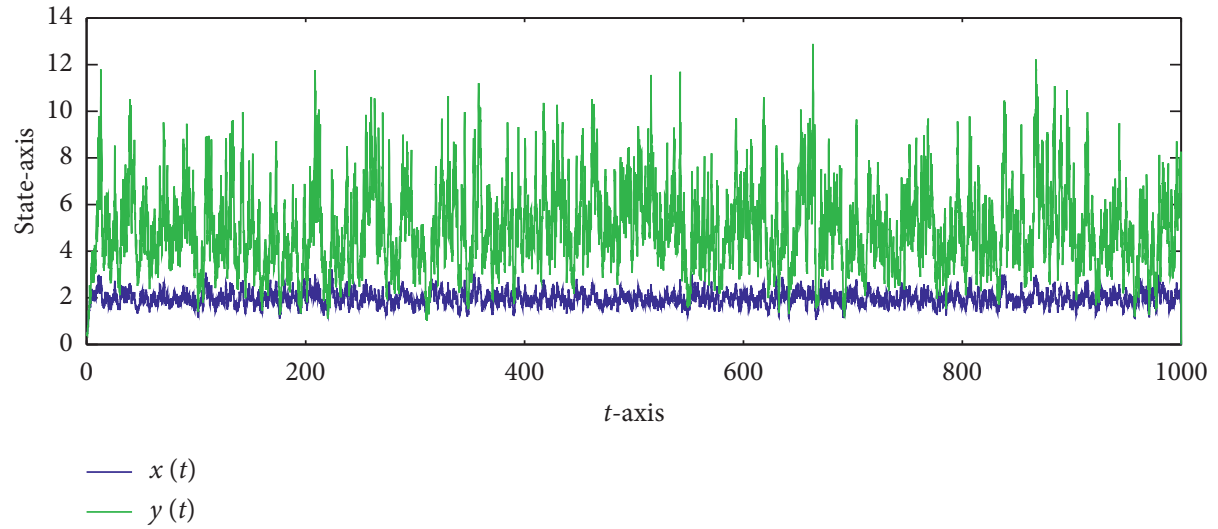

FIgURE 5: The stochastic permanence of (39) and (40) with $\pi=(0.4,0.6)$. 
We let $r_{1}(1)=0.8, b_{1}(1)=0.2, c_{1}(1)=0.8, \sigma_{1}(1)=$ $0.3 ; r_{2}(1)=0.5, b_{2}(1)=0.8, c_{2}(1)=1, \sigma_{2}(1)=0.4 ; r_{1}(2)$ $=0.7, b_{1}(2)=0.3, c_{1}(2)=0.8, \sigma_{1}(2)=0.2 ;$ and $r_{2}(2)=$ $0.4, b_{2}(2)=0.3, c_{2}(2)=1, \sigma_{2}(1)=0.4$. By Corollary 1 , we know that (39) and (40) both have stationary distribution, see Figure 2.

Suppose the distribution of $\alpha(t)$ is $\pi=(0.4,0.6)$ (see Figure 3$)$. It is easy to verify that $\zeta>0$. Theorem 1 implies that (2) has stationary distribution, see Figure 4.

If $r_{2}(1)=-0.2, r_{2}(2)=-0.3$, and $\pi=(0.4,0.6)$, then Theorem 2 shows that (39) and (40) are stochastic permanence, see Figure 5.

If $\sigma_{1}(2)=1.2, \sigma_{2}(2)=1$, and $\pi=(0.1,0.9)$, then the switching system is not stochastic permanence, but if we take $\pi=(0.9,0.1)$, then the system is stochastic permanence, which implies the switching is very important to make (39) and (40) be permanent, see Figure 1.

\section{Conclusions and Discussion}

In this paper, we study a stochastic predator-prey system with regime switching and Holling-type II functional responses. Theorems 1 and 2 give the sufficient conditions of stationary distribution and the stochastic permanence of (2). Finally, some examples are given to verify the main results. Our numerical examples reveal that switching and random factors bring much influence to the dynamics of this system.

By comparison analysis, we give Remarks 1 and 2 to show that our main results improve or generalize the corresponding results in [3]. The main method applied in this paper is by constructing some suitable functionals instead of stochastic analysis techniques to study the stationary distribution, which is less applied for switching system. In the process of our analysis, Holling-type II functional response brings some difficulties and we apply inequality techniques to overcome them.

As Arditi and Ginzburg [23] pointed out that the predator-dependent functional response can provide better description in some cases, then how to deal with predatordependent functional response such as Beddington-DeAngelis type? Furthermore, studies have shown that the mental state of the adolescent prey can be mediated by fear induced from predators and the alternation causes deleterious outcomes on their adult's survival [24] and then how fear will impact our system? All these are interesting for us to study in the future.

\section{Data Availability}

No data were used to support the findings of this study.

\section{Conflicts of Interest}

The authors declare that they have no conflicts of interest regarding the publication of this paper.

\section{References}

[1] Y. Li and D. Xiao, "Bifurcations of a predator-prey system of Holling and Leslie types," Chaos, Solitons \& Fractals, vol. 34, no. 2, pp. 606-620, 2007.

[2] D. Jiang, W. Zuo, T. Hayat, and A. Alsaedi, "Stationary distribution and periodic solutions for stochastic HollingLeslie predator-prey systems," Physica A: Statistical Mechanics and Its Applications, vol. 460, pp. 16-28, 2016.

[3] Q. Liu, L. Zu, and D. Jiang, "Dynamics of stochastic predatorprey models with Holling II functional response," Communications in Nonlinear Science and Numerical Simulation, vol. 37, pp. 62-76, 2016.

[4] C. Ji and D. Jiang, "Dynamics of a stochastic density dependent predator-prey system with Beddington-DeAngelis functional response," Journal of Mathematical Analysis and Applications, vol. 381, no. 1, pp. 441-453, 2011.

[5] H. Qiu, M. Liu, K. Wang, and Y. Wang, "Dynamics of a stochastic predator-prey system with Beddington-DeAngelis functional response," Applied Mathematics and Computation, vol. 219, no. 4, pp. 2303-2312, 2012.

[6] S.-B. Hsu and T.-W. Huang, "Global stability for a class of predator-prey systems," SIAM Journal on Applied Mathematics, vol. 55, no. 3, pp. 763-783, 1995.

[7] Y. Shao and Y. Li, "Dynamical analysis of a stage structured predator-prey system with impulsive diffusion and generic functional response," Applied Mathematics and Computation, vol. 220, pp. 472-481, 2013.

[8] R. Z. Khas'minskii, Stochastic Stability of Differential Equations, Sijthoff Noordhoff, Alphen aan den Rijn, Netherlands, 1980.

[9] W. Zuo, D. Jiang, X. Sun, T. Hayat, and A. Alsaedi, "Longtime behaviors of a stochastic cooperative Lotka-Volterra system with distributed delay," Physica A: Statistical Mechanics and Its Applications, vol. 506, pp. 542-559, 2018.

[10] X. Mao, G. Marion, and E. Renshaw, "Environmental Brownian noise suppresses explosions in population dynamics," Stochastic Processes and Their Applications, vol. 97, no. 1, pp. 95-110, 2002.

[11] X. Li, X. Mao, and X. Mao, "Population dynamical behavior of non-autonomous Lotka-Volterra competitive system with random perturbation," Discrete \& Continuous Dynamical Systems-A, vol. 24, no. 2, pp. 523-545, 2009.

[12] N. H. Du and V. H. Sam, "Dynamics of a stochastic LotkaVolterra model perturbed by white noise," Journal of Mathematical Analysis and Applications, vol. 324, no. 1, pp. 82-97, 2006.

[13] D. Jiang, Q. Zhang, T. Hayat, and A. Alsaedi, "Periodic solution for a stochastic non-autonomous competitive LotkaVolterra model in a polluted environment," Physica A: Statistical Mechanics and Its Applications, vol. 471, pp. 276287, 2017.

[14] Y. Zhao, S. Yuan, and T. Zhang, "Stochastic periodic solution of a non-autonomous toxic-producing phytoplankton allelopathy model with environmental fluctuation," Communications in Nonlinear Science and Numerical Simulation, vol. 44, pp. 266-276, 2017.

[15] Y. F. Shao, Y. Chen, and B. X. Dai, "Dynamical analysis and optimal harvesting of a stochastic three-species cooperative system with delays and Lévy jumps," Advances in Differential Equations, vol. 2018, p. 423, 2018.

[16] X. Mao and C. Yuan, Stochastic Differential Equations with Markovian Switching, Imperial College Press, London, UK, 2006. 
[17] Q. Luo and X. Mao, "Stochastic population dynamics under regime switching," Journal of Mathematical Analysis and Applications, vol. 334, no. 1, pp. 69-84, 2007.

[18] L. Zu, D. Jiang, and D. O’Regan, "Conditions for persistence and ergodicity of a stochastic Lotka-Volterra predator-prey model with regime switching," Communications in Nonlinear Science and Numerical Simulation, vol. 29, no. 1-3, pp. 1-11, 2015.

[19] J. Bao and J. Shao, "Permanence and extinction of regimeswitching predator-prey models," SIAM Journal on Mathematical Analysis, vol. 48, no. 1, pp. 725-739, 2016.

[20] H. Chen, P. Shi, and C.-C. Lim, "Stability analysis for neutral stochastic delay systems with Markovian switching," Systems \& Control Letters, vol. 110, pp. 38-48, 2017.

[21] M. Liu, X. He, and J. Yu, "Dynamics of a stochastic regimeswitching predator-prey model with harvesting and distributed delays," Nonlinear Analysis: Hybrid Systems, vol. 28, pp. 87-104, 2018.

[22] S. Wang, L. Wang, and T. Wei, "Permanence and asymptotic behaviors of stochastic predator-prey system with Markovian switching and Lévy noise," Physica A: Statistical Mechanics and Its Applications, vol. 495, pp. 294-311, 2018.

[23] R. Arditi and L. R. Ginzburg, "Coupling in predator-prey dynamics: ratio-dependence," Journal of Theoretical Biology, vol. 139, no. 3, pp. 311-326, 1989.

[24] A. Das and G. P. Samanta, "Modelling the fear effect in a twospecies predator-prey system under the influence of toxic substances," Rendiconti del Circolo Matematico di Palermo Series, vol. 2, 2020.

[25] D. J. Higham, "An algorithmic introduction to numerical simulation of stochastic differential equations," SIAM Review, vol. 43, no. 3, pp. 525-546, 2001. 